\title{
Phosphoproteomics Provides Novel Insights into the Response of Primary Acute Lymphoblastic Leukemia Cells to Microtubule Depolymerization in G1 Phase of the Cell Cycle
}

Magdalena Delgado, Charity L. Washam, Alicja Urbaniak, Billie Heflin, Aaron J. Storey, Renny S. Lan, Samuel G. Mackintosh, Alan J. Tackett, Stephanie D. Byrum,* and Timothy C. Chambers*

Cite This: ACS Omega 2021, 6, 24949-24959

Read Online

ABSTRACT: Microtubule targeting agents (MTAs) have been used for the treatment of cancer for many decades and are among the most successful chemotherapeutic agents. However, their application and effectiveness are limited because of toxicity and resistance as well as a lack of knowledge of molecular mechanisms downstream of microtubule inhibition. Insights into key pathways that link microtubule disruption to cell death is critical for optimal use of these drugs, for defining biomarkers useful in patient stratification, and for informed design of drug combinations. Although MTAs characteristically induce death in mitosis, microtubule destabilizing agents such as vincristine also induce death directly in G1 phase in primary acute lymphoblastic

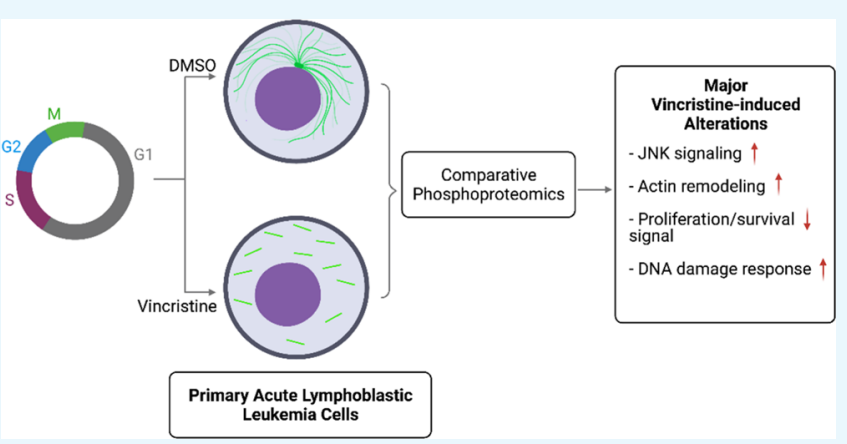
leukemia (ALL) cells. Because many signaling pathways regulating cell survival and death involve changes in protein expression and phosphorylation, we undertook a comprehensive quantitative proteomic study of G1 phase ALL cells treated with vincristine. The results revealed distinct alterations associated with c-Jun $\mathrm{N}$-terminal kinase signaling, anti-proliferative signaling, the DNA damage response, and cytoskeletal remodeling. Signals specifically associated with cell death were identified by pre-treatment with the CDK4/6 inhibitor palbociclib, which caused G1 arrest and precluded death induction. These results provide insights into signaling mechanisms regulating cellular responses to microtubule inhibition and provide a foundation for a better understanding of the clinical mechanisms of MTAs and for the design of novel drug combinations. The mass spectrometry proteomics data have been deposited to the PRIDE Archive (http://www.ebi.ac.uk/pride/archive/) via the PRIDE partner repository with the data set identifier PXD027190 and 10.6019/PXD027190.

\section{INTRODUCTION}

Drugs that target tubulin and microtubules represent an important class of cancer chemotherapeutic agents. ${ }^{1-3}$ The most widely utilized microtubule targeting agents (MTAs) clinically are the vinca alkaloids including vinblastine and vincristine $(\mathrm{VCR})^{4,5}$ and the taxanes including paclitaxel and docetaxel. ${ }^{6,7}$ The vinca alkaloids are primarily used to treat leukemia and lymphoma and certain solid tumors including lung cancer. ${ }^{3,4}$ They act by binding to tubulin dimers and promote microtubule depolymerization. ${ }^{8}$ In laboratory cell lines, these effects typically manifest during mitosis resulting in mitotic arrest and cell death. ${ }^{9-13}$

Despite their success, toxicity is a major limiting factor because MTAs affect cell division in normal cells, which causes myelosuppression, and they inhibit microtubule function in neuronal cells, which causes neuropathies. ${ }^{14-16}$ This has led to the testing of inhibitors of mitotic kinases, with the rationale that such agents would promote mitotic death in cancer cells but spare non-dividing neuronal cells. ${ }^{17}$ However, in clinical trials mitotic kinase inhibitors have shown poor activity and unacceptable toxicity. ${ }^{18,19}$ This in turn has led to the suggestion that the clinical success of MTAs may not be because of effects on the mitotic spindle but instead may be due to impairment of interphase microtubule functions. ${ }^{18-20}$ Supporting this notion are long-established observations that MTAs are effective clinically even for human tumors with protracted doubling times and low mitotic indices. ${ }^{21}$

While interference with interphase microtubule functions may be a major factor in the therapeutic efficacy of MTAs in the clinical setting, it has been difficult to test this experimentally because most laboratory cell lines only show susceptibility to MTAs during mitosis. Recently, however, we

Received: July 23, 2021

Published: September 16, 2021 
reported that primary cultures of ALL cells are susceptible to microtubule depolymerizing drugs such as VCR not only in mitosis but also in the interphase, specifically during G1 phase. $^{22}$ We define MTA-mediated interphase death as cell death in interphase in response to perturbations of interphase microtubules caused by an MTA. This is in contrast to death in interphase following an aberrant mitosis induced by an MTA. In the former case, damage and death occur de novo in interphase, whereas in the latter case, damage occurs in mitosis but manifests later in subsequent interphase after mitotic slippage. ${ }^{23-25}$

Mitotic death typically occurs via intrinsic apoptosis, and our previous studies have shown that this is regulated, at least in part, by phosphorylation and inactivation of pro-survival members of the Bcl-2 family of proteins. ${ }^{26-28}$ In preliminary studies, we have discovered that G1 phase death of primary ALL cells in response to VCR occurs via a distinct pathway that is independent of $\mathrm{Bcl}-2$ proteins. Many cell survival and cell death pathways involve changes in the expression levels and phosphorylation of specific regulatory proteins. The protein kinase $\mathrm{PKB} / \mathrm{AKT}$, for example, is a key mediator of survival signaling through the phosphorylation and inactivation of pro-apoptotic target proteins. ${ }^{29}$ Similarly, several cell death pathways signal via mechanisms resulting in protein degradation and/or phosphorylation. For example, mitotic death is strongly associated with phosphorylation and degradation of the survival protein $\mathrm{Mcl}-1,{ }^{30}$ and cell death via necroptosis is mediated by phosphorylation and activation of mixed lineage kinase domain-like pseudokinase by receptor-interacting kinase $3 .^{31}$

In order to investigate key pathways involved in VCRmediated G1 phase death in primary ALL cells, we undertook a comprehensive quantitative proteomic and phosphoproteomic study. This analysis revealed that major alterations in response to drug treatment included activation of the c-Jun $\mathrm{NH}_{2}$ terminal kinase (JNK) pathway, activation of the DNA damage response (DDR), downregulation of several proliferation and survival signaling pathways, and cytoskeletal remodeling. Signals specifically associated with cell death were identified by inducing G1 phase arrest which precluded death induction by VCR. These results provide novel insights into signaling mechanisms regulating cellular responses to microtubule inhibition during interphase and a foundation for investigating mechanisms underlying the clinical actions of MTAs.

\section{RESULTS AND DISCUSSION}

Establishment of Experimental Conditions for Optimal Sampling. Our previous studies have shown that when primary ALL cells isolated in G1 phase are treated with microtubule destabilizers such as VCR, they die directly, ${ }^{22,32}$ through pathways that remain to be fully defined. Microtubule destabilizers can be envisioned to exert their action through several steps including penetration into the cell, microtubule depolymerization, initiation of appropriate cellular responses that signal such perturbations, and eventual cell death. Because the focus of this study was to identify changes in protein and phosphoprotein expression that act as signals for cell death induction, it was important to select a treatment time that was sufficient for anticipated signal generation but which preceded cell death. Therefore, primary ALL cells in G1 phase, enriched to $98 \%$ by centrifugal elutriation, ${ }^{33}$ were treated with vehicle [0.1\% dimethyl sulfoxide (DMSO)] or $100 \mathrm{nM} \mathrm{VCR} \mathrm{for}$ periods from 8-24 h. Cell extracts were probed for expression of poly (ADP-ribose) polymerase (PARP), which is characteristically cleaved during cell death. As shown in Figure 1A, PARP remained intact with vehicle treatment but underwent progressive cleavage after VCR treatment, with partial cleavage by $16 \mathrm{~h}$ and full cleavage by $24 \mathrm{~h}$.

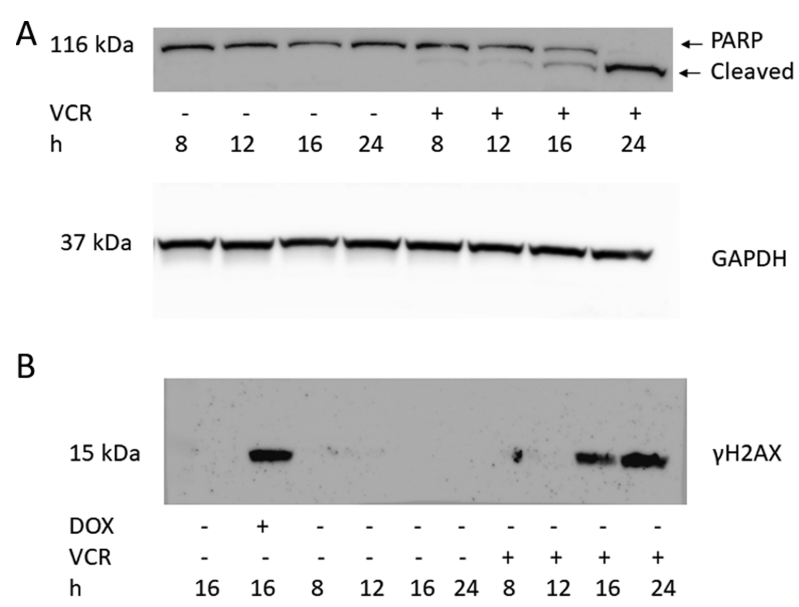

Figure 1. Kinetics of cell death. Primary ALL cells in G1 phase were prepared by centrifugal elutriation as described in the Experimental Section and treated with vehicle (0.1\% DMSO) or $100 \mathrm{nM}$ VCR for the times indicated. (A) Extracts were prepared and subjected to immunoblot analysis for PARP, with GAPDH as a loading control. The molecular masses of intact PARP and GAPDH are indicated on the left; cleaved PARP has a molecular mass of about $85 \mathrm{kDa}$. (B) Samples from panel A, together with extracts from ALL cells treated with vehicle or $1 \mu \mathrm{M}$ doxorubicin (DOX) for $16 \mathrm{~h}$, were subjected to immunoblot analysis for $\gamma \mathrm{H} 2 \mathrm{AX}$.

To further support these observations on the kinetics of cell death, cell extracts were also immunoblotted for $\gamma \mathrm{H} 2 \mathrm{AX}$, a phosphorylated histone variant that serves as a marker for the DDR involving double-strand breaks. ${ }^{34}$ As shown in Figure 1B, VCR induced $\gamma \mathrm{H} 2 \mathrm{AX}$ at $16 \mathrm{~h}$ and $24 \mathrm{~h}$ of VCR treatment, whereas no signal was observed in DMSO-treated cells. The anticancer drug DOX, which is known to cause DNA doublestrand breaks, served as a positive control. Importantly however, no $\gamma \mathrm{H} 2 \mathrm{AX}$ signal was observed in cells treated with VCR for $12 \mathrm{~h}$ (Figure 1B). Based on these results, an optimal pre-death treatment time of $12 \mathrm{~h}$ was selected for the samples to be analyzed by mass spectrometry.

Proteomic and Phosphoproteomic Changes with VCR Treatment. The relative abundance of proteins in vehicle- and VCR-treated (VCR) G1 phase ALL cells was determined by LC-MS/MS as described in the Experimental Section. The full list is shown in Table S1. A total of 7525 proteins were identified but only one protein, the AP-1 transcription factor protein c-Jun, was significantly differentiated between VCR and vehicle with an absolute fold change $>2$ and a false discovery rate (FDR) adjusted $p$-value < 0.05 , as shown in the volcano plot in Figure $2 \mathrm{~A}$. In contrast to the low incidence of changes in protein abundance, several changes in phosphoprotein abundance occurred when vehicleand VCR-treated G1 phase ALL cells were compared, with the majority of phosphopeptides being increased with drug treatment. The full list is shown in Table S2. A total of 4499 phosphorylated peptides were identified, and 92 phosphosites were found to be differentially regulated between VCR and vehicle including 82 sites with increased expression after VCR 


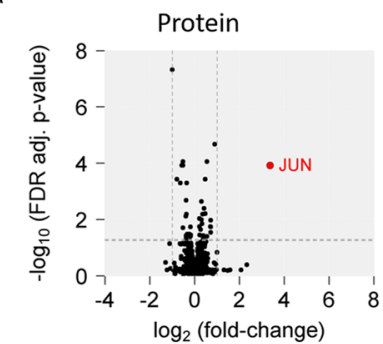

C

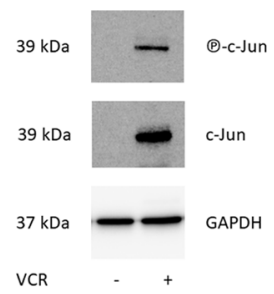

B
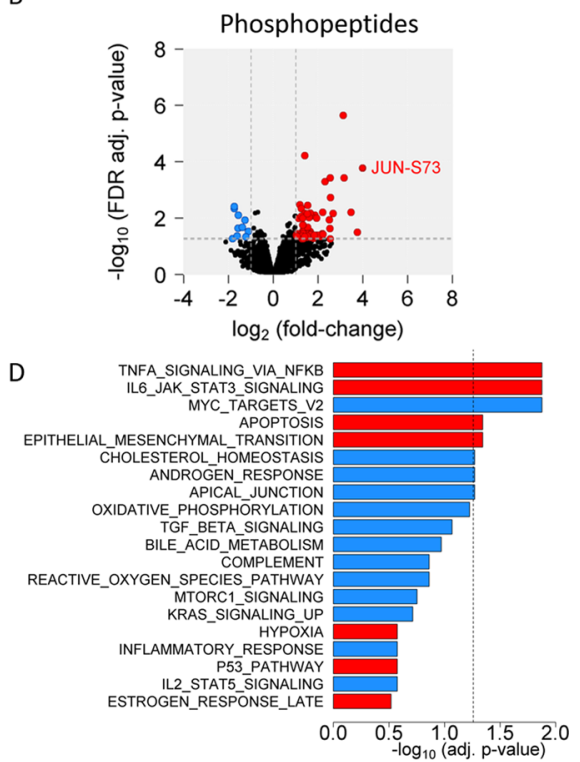

Figure 2. Phosphoproteomic changes associated with VCR treatment. ALL cells in G1 phase were treated with $0.1 \%$ DMSO or $100 \mathrm{nM}$ VCR for $12 \mathrm{~h}$ and samples were prepared for analysis as described in the Experimental Section. (A) Volcano plot of protein significant abundance in VCR compared to DMSO treatment. (B) Volcano plot of phosphopeptide significant abundance. $X$-axis indicates $\log 2$ fold change. $Y$-axis indicates $-\log 10$ FDR-adjusted $p$-value. Proteins or phosphopeptides shown in the upper right quadrant in red are significant by FDR-adjusted $p$-value $<$ 0.05 and $\log 2$ fold change $>1$. The upper left quadrant indicates features significant by FDR-adjusted $p$-value $<0.05$ and log 2 fold change $<-1$ shown in blue. Jun and Jun S73 were significant as indicated on the volcano plots. (C) Immunoblot validation. Extracts from G1 phase ALL cells untreated or treated with $100 \mathrm{nM}$ VCR for $12 \mathrm{~h}$ were subjected to immunoblot analysis for c-Jun, phospho(Ser73)-c-Jun, or GAPDH, as indicated. (D) Barplot of the top 20 Hallmark gene sets identified based on the protein analysis comparing VCR to DMSO treatment. Pathway up-regulation is shown in red, while pathway down-regulation is shown in blue. $X$-axis indicates the $-\log 10$ FDR-adjusted $p$-value, and values $>1.3$ indicate significant pathway expression.

Table 1. VCR Increases Phosphorylation of Several Proteins Associated with the Cytoskeleton and Its Regulation ${ }^{a}$

\begin{tabular}{|c|c|c|c|c|c|c|}
\hline uniprotID & description & gene name & $\begin{array}{l}\text { amino acid and } \\
\text { position }\end{array}$ & $\begin{array}{l}\log 2 \text { fold } \\
\text { change }\end{array}$ & $P$-value & $\begin{array}{l}\text { FDR adj } \\
P \text {-value }\end{array}$ \\
\hline Q99426 & Tubulin-folding cofactor B & TBCB & S110 & 2.54 & $2.9 \times 10^{-6}$ & $1.9 \times 10^{-3}$ \\
\hline E7EVA0 & microtubule-associated protein & MAP4 & S2290 & 1.79 & $1.6 \times 10^{-3}$ & $5.2 \times 10^{-2}$ \\
\hline Q63ZY3 & $\begin{array}{l}\mathrm{KN} \text { motif and ankyrin repeat } \\
\text { domain-containing protein } 2\end{array}$ & KANK2 & S463 & 1.72 & $7.9 \times 10^{-4}$ & $4.0 \times 10^{-2}$ \\
\hline MOR0P8 & unconventional myosin-IXb & MYO9B & T1346 & 3.12 & $5.0 \mathrm{E}-10$ & $2.2 \times 10^{-6}$ \\
\hline Q9UDT6 & CAP-Gly domain-containing linker protein 2 & CLIP2 & S211 & 2.54 & $4.1 \times 10^{-7}$ & $3.7 \times 10^{-4}$ \\
\hline Q16643 & drebrin & DBN1 & T346 & 1.53 & $7.1 \times 10^{-6}$ & $3.5 \times 10^{-3}$ \\
\hline Q99426 & tubulin-folding cofactor B & TBCB & S123 & 1.59 & $5.6 \times 10^{-5}$ & $9.4 \times 10^{-3}$ \\
\hline F8VZN8 & $\begin{array}{l}\text { protein phosphatase } 1 \text { regulatory subunit } 12 \mathrm{~A} \\
\text { (Fragment) }\end{array}$ & PPP1R12A & S299 & 3.74 & $3.6 \times 10^{-4}$ & $3.1 \times 10^{-2}$ \\
\hline Q9Y6G9 & cytoplasmic dynein 1 light intermediate chain 1 & DYNC1LI1 & S516 & 2.21 & $5.9 \times 10^{-4}$ & $3.7 \times 10^{-2}$ \\
\hline P42331 & Rho GTPase-activating protein 25 & ARHGAP25 & S533 & 2.54 & $1.7 \times 10^{-3}$ & $5.4 \times 10^{-2}$ \\
\hline
\end{tabular}

${ }^{a_{T}}$ Ten of the phosphopeptides related to the cytoskeleton that were significantly differentiated in VCR-treated versus DMSO-treated cells are shown.

treatment (Figure 2B). One of the most significant upregulated phosphosites was c-Jun S73 (Figure 2B). This is a major site of phosphorylation in the transactivation domain of c-Jun by c-Jun N-terminal kinases, JNK. ${ }^{35}$ The observation of increased c-Jun protein expression concomitant with increased c-Jun S73 phosphorylation is consistent with established mechanisms of c-Jun induction, since the c-Jun promoter contains two AP-1 sites that are responsive to activation by S73-phosphorylated c-Jun, resulting in a strong auto-amplifying loop. ${ }^{36}$ The increase in c-Jun and in c-Jun S73 in the VCRtreated G1 phase ALL cells was confirmed by immunoblot analysis (Figure 2C). Importantly, our previous studies have shown that VCR caused increased expression of c-Jun in HeLa cells. ${ }^{37}$ However, it was in the context of mitotic death, not death in G1 phase that occurred here. Thus, the present findings establish c-Jun induction as a general cellular response to VCR regardless of the cell cycle phase in which the drug acts.

A gene set enrichment analysis was performed from the protein abundance data, which essentially revealed pathways regulated or mediated by c-Jun since this was the lone major alteration induced by VCR. These included TNF $\alpha$ signaling via $\mathrm{NF} \kappa \mathrm{B}, \mathrm{IL} 6 / \mathrm{JAK} / \mathrm{STAT} 3$ signaling, and changes related to the epithelial-mesenchymal transition, all of which were upregulated (Figure 2D). In addition, apoptosis signaling was upregulated, consistent with the well-established role that the JNK/c-Jun pathway plays in regulation of apoptosis. ${ }^{38}$ Interestingly, a subset of Myc target genes was significantly 
Table 2. PHOXTRACK Analysis of Significant Phosphopeptides Differentially Regulated in VCR-Treated (100 nM, 12 h) Versus Vehicle (0.1\% DMSO) G1 phase ALL Cells ${ }^{a}$

\begin{tabular}{|c|c|c|c|c|c|c|c|c|}
\hline kinase & database & no. of phosphosites & EV & $\mathrm{NEV}$ & $p$-value & FDR $p$-value & PHOXTRACK score & DDR \\
\hline DNA-PK & PSP & 5 & 0.80 & 2.24 & 0.0008 & 0.0318 & 2.17 & $\mathrm{X}$ \\
\hline JNK2 & PSP & 3 & 0.95 & 2.09 & 0.0004 & 0.0358 & 2.02 & \\
\hline ATR & PSP & 7 & 0.65 & 2.11 & 0.0024 & 0.0455 & 2.02 & $\mathrm{x}$ \\
\hline ATM & PSP & 7 & 0.61 & 2.00 & 0.0040 & 0.0439 & 1.91 & $\mathrm{x}$ \\
\hline PLK3 & PSP & 4 & 0.80 & 2.01 & 0.0037 & 0.0500 & 1.91 & $\mathrm{x}$ \\
\hline PRKDC & HPRD & 3 & 0.90 & 1.98 & 0.0024 & 0.0359 & 1.91 & $\mathrm{x}$ \\
\hline PRKDC & Swiss & 3 & 0.90 & 1.99 & 0.0019 & 0.0402 & 1.91 & \\
\hline JNK1 & PSP & 7 & 0.58 & 1.91 & 0.0084 & 0.0529 & 1.81 & \\
\hline MAPK14 & Swiss & 3 & 0.84 & 1.87 & 0.0071 & 0.0649 & 1.75 & \\
\hline ATM & Swiss & 5 & 0.62 & 1.73 & 0.0222 & 0.1328 & 1.50 & $\mathrm{x}$ \\
\hline GSK-3 $\beta$ & PELM & 3 & 0.78 & 1.72 & 0.0206 & 0.1295 & 1.50 & \\
\hline $\mathrm{CSNK} 2 \mathrm{~A} 2$ & HPRD & 13 & 0.39 & 1.69 & 0.0296 & 0.1398 & 1.46 & \\
\hline ERK1 & PSP & 18 & -0.30 & -1.52 & 0.0646 & 0.1745 & -1.25 & \\
\hline $\mathrm{CDK} 2$ & PSP & 43 & -0.21 & -1.58 & 0.0490 & 0.1365 & -1.37 & \\
\hline LYN & HPRD & 3 & -0.75 & -1.66 & 0.0300 & 0.0974 & -1.50 & \\
\hline MAPK1 & HPRD & 8 & -0.49 & -1.72 & 0.0258 & 0.0778 & -1.58 & \\
\hline SRC & PSP & 5 & -0.70 & -1.95 & 0.0050 & 0.0186 & -1.91 & \\
\hline MAPK3 & HPRD & 7 & -0.60 & -1.95 & 0.0058 & 0.0197 & -1.92 & \\
\hline $\mathrm{ABL}$ & PSP & 3 & -0.90 & -2.00 & 0.0014 & 0.0154 & -1.97 & \\
\hline $\mathrm{ABL} 1$ & Swiss & 3 & -0.90 & -2.00 & 0.0016 & 0.0164 & -1.97 & \\
\hline LCK & PSP & 4 & -0.83 & -2.09 & 0.0013 & 0.0089 & -2.07 & \\
\hline EGFR & PELM & 4 & -0.87 & -2.19 & 0.0006 & 0.0042 & -2.18 & \\
\hline MET & PELM & 4 & -0.87 & -2.19 & 0.0005 & 0.0047 & -2.18 & \\
\hline MET & HPRD & 4 & -0.87 & -2.20 & 0.0003 & 0.0052 & -2.19 & \\
\hline INSR & PSP & 4 & -0.91 & -2.28 & 0.0002 & 0.0031 & -2.28 & \\
\hline INSR & PELM & 4 & -0.94 & -2.36 & 0.0001 & 0.0019 & -2.35 & \\
\hline EGFR & PSP & 5 & -0.87 & -2.43 & 0.0001 & 0.0018 & -2.43 & \\
\hline EGFR & HPRD & 5 & -0.87 & -2.43 & 0.0000 & 0.0012 & -2.43 & \\
\hline INSR & HPRD & 5 & -0.90 & -2.52 & 0.0000 & 0.0017 & -2.51 & \\
\hline
\end{tabular}

${ }^{a}$ The database column indicates whether the kinase was identified in the PSP, HPRD, Swiss-Prot, or PELM database. EV is the enrichment value, and NEV is the normalized enrichment value for each kinase, which can be used to compare the activities of kinases between samples. The $p$-value is based on a Kolomogorov-Smirnov sum statistic. The PHOXTRACK score combines the directionality of the NEV (activated vs inhibited) and the magnitude of the false discovery rate (FDR) $p$-value. The DDR column indicates kinases involved in DDRs. ${ }^{44}$

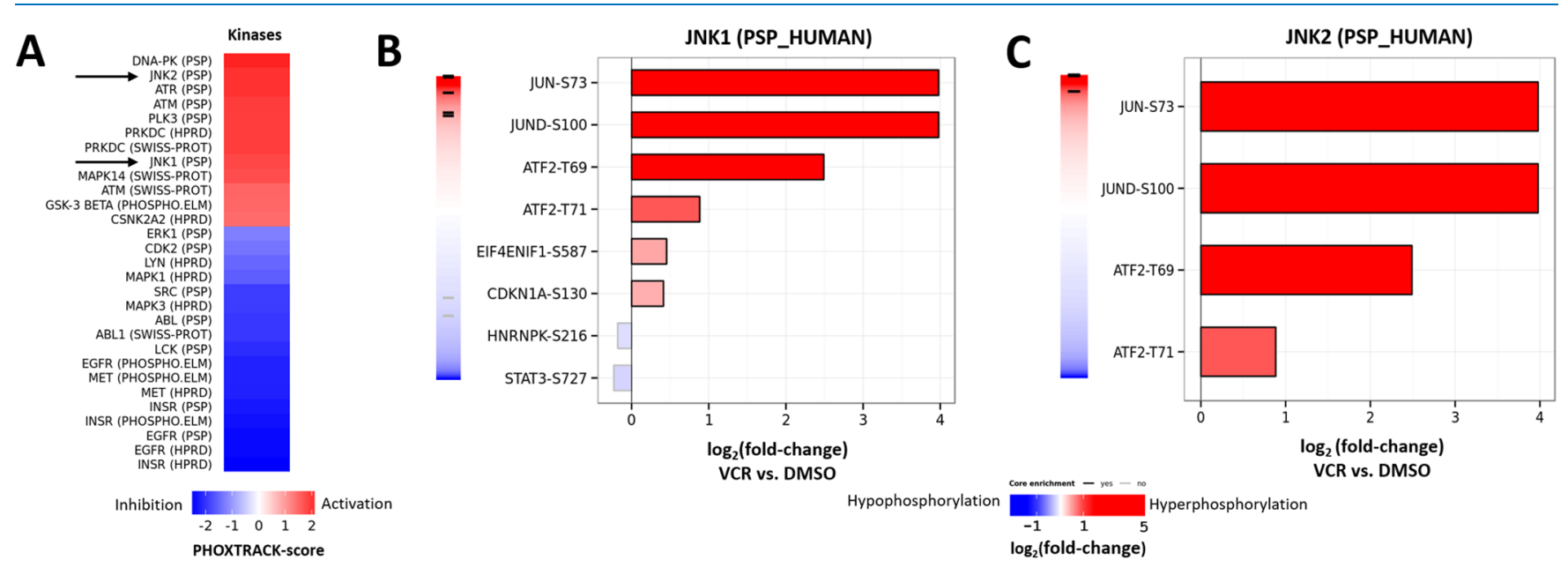

Figure 3. PHOXTRACK prediction of kinase substrate activity. (A) Significantly differentiating phosphopeptide sequences from the VCR to DMSO comparison were analyzed using PHOXTRACK. The phosphopeptides were compared to phosphosite databases, including PhosphoSitePlus (PSP), Swiss-Prot, Human Protein Reference Database (HPRD), and Phospho.ELM (PELM), which contain thousands of substrate sequence/kinase interactions. An enrichment score reflects the degree to which the kinase-specific set of phosphosites is over represented at the top (red, activated) or bottom (blue, inhibited). JNK1 and JNK2 which are highlighted in panels B and C are indicated by arrows. (B,C) PHOXTRACK substrate phosphorylation view displays the phosphorylated substrates of JNK1 (B) and JNK2 (C). Hyperphosphorylated substrates after VCR treatment are indicated in red, while hypophosphorylated substrates are indicated in blue. 
downregulated under these conditions (Figure 2D), which may be related to the reported ability of JNK to phosphorylate Myc, causing its ubiquitination and degradation. ${ }^{39}$ Consistent with this, Myc protein abundance significantly decreased after VCR treatment (Table S1).

VCR Promotes Cytoskeletal Alterations. VCR treatment of G1 phase ALL cells resulted in increased phosphorylation of several cytoskeletal and cytoskeletalregulating proteins. These are listed in Table 1 together with their UniProtIDs, from which details of their function can be accessed, the phospho-site in question, and its change of abundance after VCR treatment relative to DMSO. Some of these alterations are consistent with microtubule destabilization and are an expected consequence of VCR treatment. For example, microtubule-associated protein (MAP) 4, which showed increased phosphorylation of S2290 (Table 1), counteracts destabilization of interphase microtubules, a function that is modulated by phosphorylation. ${ }^{40}$ Similarly, tubulin folding cofactor B (S110 and S123) is involved in tubulin heterodimer dissociation and phosphorylation of these sites promotes this property, and CLIP2 and DYNC1L11 (Table 1) are involved in microtubule-based movement. ${ }^{40}$ Of significant interest, and in addition to changes associated with microtubule structure, there were a number of phosphorylative modifications indicative of actin remodeling (Table 1). These included the protein phosphatase PPR1R12A (S299, 13-fold increase), which regulates the interaction of actin and myosin; unconventional myosin-IXB, which is involved in actin-based movement and showed an 8-fold increase in T1356 phosphorylation; Rho GTPase-activating protein 25 (S533), which regulates actin filament organization; KANK2 (S463), which has several functions including a role in actin stress fiber formation; $^{41}$ and drebrin (T346), which is an actin cytoskeleton reorganizing protein. ${ }^{42}$ These results are intriguing and suggest that a major response to microtubule depolymerization in interphase in ALL cells is remodeling of the actin cytoskeleton. This may occur as a compensatory response and an attempt by the cell to employ actin to correct cytoskeletal deficiencies caused by loss of microtubule filaments. It would be of significant interest to determine whether other cell types respond similarly and to determine whether compounds that target actin, which have been described, ${ }^{43}$ synergize with MTAs in killing ALL cells in G1 phase.

Prediction of Kinase-Substrate Relationships. Prediction of kinase-substrate relationships are shown in Table 2 and Figure 3. Differentially abundant phosphorylated sites were analyzed using PHOsphosite-X-TRacing Analysis of Causal Kinases (PHOXTRACK) to identify putative protein kinases and their substrates. The phosphopeptide-flanking regions were used for the analysis, which included the modified amino acid, plus and minus six amino acids on either side of the phospho-site. From the 92 phosphorylated sites, the protein kinases DNA-PK, JNK1, JNK2, ATR, and ATM were among the predicted kinases activated with VCR treatment in G1 phase ALL cells (Figure 3A). JNK1 and JNK2 bind and phosphorylate sites in the transactivation domain of c-Jun, including S73, ${ }^{35}$ supporting the findings described above of cJun S73 phosphorylation in VCR-treated cells.

JNK1 and JNK2 also phosphorylate the related AP-1 transcription factor, JunD, at S100, and the ATF-2 transcription factor at $\mathrm{T} 69$ and $\mathrm{T} 71$, sites within the transactivation domain. $^{35}$ These were also found to be significantly phosphorylated in response to VCR treatment (Figure $3 \mathrm{~B}, \mathrm{C})$. Overall, the results demonstrate JNK signaling as a prominent response to VCR in G1 phase ALL cells. This conclusion is consistent with earlier studies, using techniques such as enzyme assays and immunoblotting, which implicated JNK activation as an important response to microtubule depolymerization during interphase. ${ }^{45}$

Of further interest, several phosphosites were less abundant after VCR treatment, reflecting downregulation of a number of proliferation- and growth-associated kinase pathways. These included mitogen-activated kinases such as ERK1 and MAPK1; the cell cycle kinase CDK2; and several receptor (EGFR) and non-receptor (LYN, SRC, ABL, and LCK) tyrosine kinases (Table 2 and Figure 3A). Thus VCR-induced death of G1 phase ALL cells is associated not only with an upregulation of apoptotic signaling but with diminished proliferation signaling.

These results also illustrate and reinforce the reciprocal nature of JNK and ERK signaling which has been established in a number of other systems. ${ }^{38}$

Several kinases activated by VCR treatment, including DNAPK, ATM, ATR, and PRKDC, are established mediators of the DDR (Table 2 and Figure 3A). It is important to note that the presence of DDR signals in the proteomic analysis was observed with samples derived from cells treated with VCR for $12 \mathrm{~h}$. In contrast, expression of $\gamma \mathrm{H} 2 \mathrm{AX}$ determined by immunoblotting, indicative of DDR, occurred at $16 \mathrm{~h}$ of treatment and was not observed at the earlier time-point of 12 $\mathrm{h}$ (Figure 1B). One explanation is that MS analysis is much more sensitive than immunoblotting, with signals detected at levels below those readily observed by immunoblotting. Further, the early DDR signals observed after $12 \mathrm{~h}$ treatment may not be a reflection of initial DNA degradation associated with impending cell death, but instead might represent a primary response to VCR. For example, it has been reported that microtubule disruption in interphase impedes the ability of cells to translocate DNA repair proteins to the nucleus, by rendering microtubule structures that normally mediate protein trafficking inoperative. ${ }^{46}$ The lack of endogenous DNA repair leads to a DDR which acts as an initial signal for cell death.

Defining Signals that Associate with Cell Death. These results indicated that VCR treatment of ALL cells in G1 phase influenced specific pathways as shown by changes in phosphorylation patterns, in particular JNK signaling, DDR, and cytoskeletal alterations. Of major interest was determining which of these events was related to cell death and which was unrelated. As indicated above, the observed DDR may be a primary signal for the initiation of cell death or may simply reflect DNA fragmentation as a consequence of cell death. Likewise, JNK signaling could be a key response regulating cell death or may play a protective or peripheral role, for example, as a response to cytoskeletal reorganization independent of cell death. Fortuitously, we have shown previously that while ALL cells in G1 phase are susceptible to VCR and undergo death, ALL cells arrested in G1 phase by palbociclib (PCB), a CDK4/ 6 inhibitor, ${ }^{47}$ are refractory to VCR and remain viable. ${ }^{48} \mathrm{We}$ have hypothesized that microtubules are essential for cells actively cycling through the G1 phase, in order for protein trafficking to occur normally to enable G1 phase advance, while non-cycling G1-arrested cells have no purpose for microtubules and hence are unaffected by $\mathrm{VCR}^{48} \mathrm{We}$ reasoned that co-treated cells should not display proteomic signaling events associated with cell death. Conversely, dual- 
A

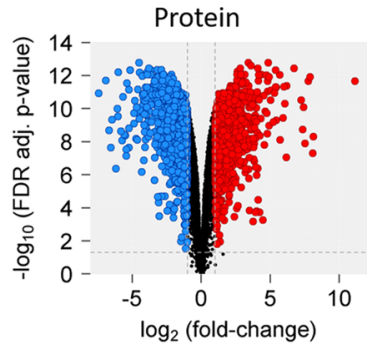

C

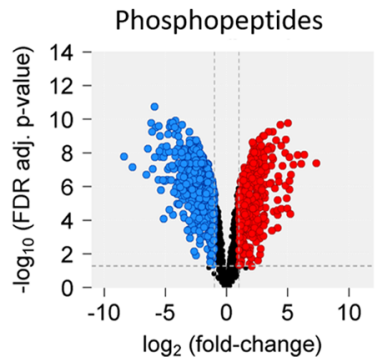

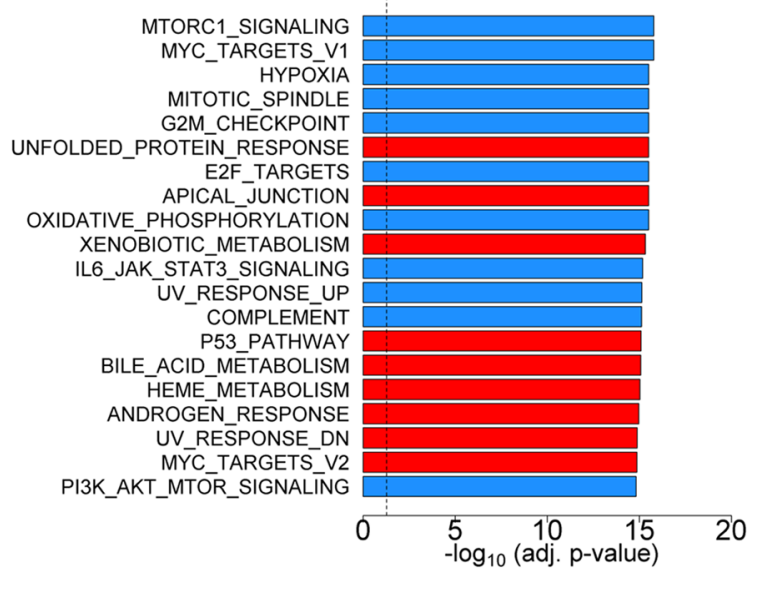

Figure 4. Phosphoproteomic changes associated with PCB treatment. ALL cells in G1 phase were treated with $0.1 \%$ DMSO or $1 \mu \mathrm{M}$ PCB for $72 \mathrm{~h}$ and samples prepared for analysis as described in the Experimental Section. (A) Volcano plot of the protein abundance differential expression. $X$ axis indicates $\log 2$ fold change. $Y$-axis indicates the $-\log 10$ FDR-adjusted $p$-value. Proteins shown in the upper right quadrant in red are significant by FDR-adjusted $p$-value $<0.05$ and $\log 2$ fold change $>1$. The upper left quadrant indicates features significant by FDR-adjusted $p$-value $<0.05$ and $\log 2$ fold change $<-1$ shown in blue. (B) Barplot of the top 20 Hallmark gene sets identified based on the protein analysis comparing VCR to DMSO treatment. Pathway up-regulation is shown in red, while pathway down-regulation is shown in blue. $X$-axis indicates the -log10 FDRadjusted $p$-value and values $>1.3$ indicate significant pathway expression. (C) Volcano plot of phosphopeptide abundance differential expression. Thresholds for red and blue quadrants are defined as in panel A.
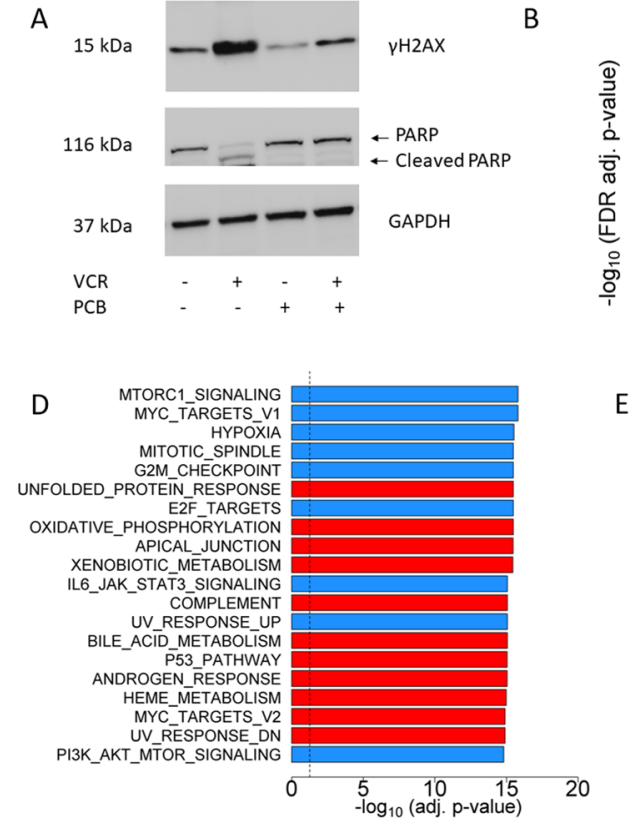

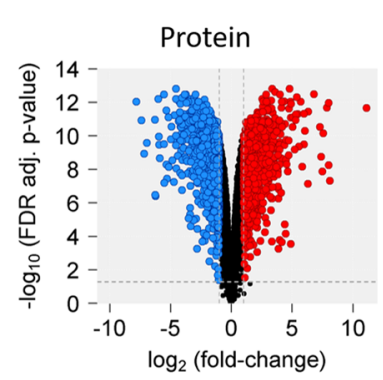

E

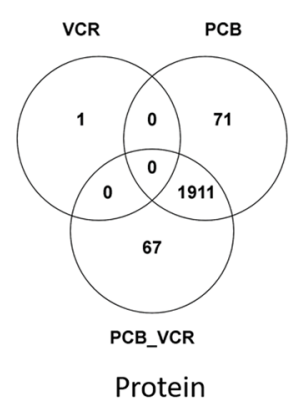

C

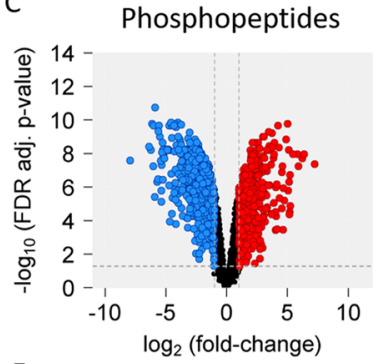

$\mathrm{F}$

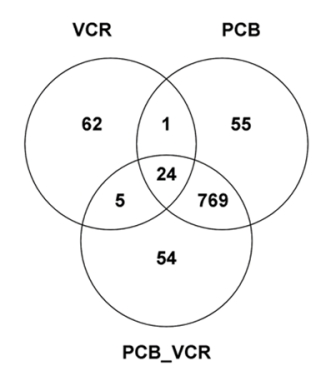

Phosphopeptides

Figure 5. Phosphoproteomic changes associated with combined VCR and PCB treatment. (A) ALL cells in G1 phase were treated with $0.1 \%$ DMSO (-) or $100 \mathrm{nM}$ VCR for $12 \mathrm{~h}$ without or with pretreatment with $1 \mu \mathrm{M}$ PCB for $72 \mathrm{~h}$. Extracts were prepared and subjected to immunoblot analysis for $\gamma \mathrm{H} 2 \mathrm{AX}$, PARP, or GAPDH, as indicated. (B) Volcano plot of protein significant abundance in PCB + VCR compared to DMSO treatment. (C) Volcano plot of phosphopeptide significant abundance. For panels B and C, $X$-axis indicates log 2 fold change. $Y$-axis indicates $-\log 10$ FDR-adjusted $p$-value. Proteins or phosphopeptides shown in the upper right quadrant in red are significant by FDR-adjusted $p$-value $<$ 0.05 and $\log 2$ fold change $>1$. The upper left quadrant indicates features significant by FDR-adjusted $p$-value $<0.05$ and $\log 2$ fold change $<-1$ shown in blue. (D) Barplot of the top 20 Hallmark gene sets identified based on the protein analysis comparing PCB + VCR to DMSO treatment. Pathway up-regulation is shown in red, while pathway down-regulation is shown in blue. $X$-axis indicates the $-\log 10$ FDR-adjusted $p$-value and values $>1.3$ indicate significant pathway expression. (E,F) Venn diagram indicates the number of significantly differentiating proteins (E) or phosphopeptides (F) that are in common or unique to the VCR versus DMSO, PCB versus DMSO, and PCB + VCR versus DMSO comparisons. Proteins and phosphopeptides are defined as significant with a threshold of FDR-adjusted $p$-value $<0.05$ and fold change $>$ or $<2$.

treated cells might still undergo signaling events unrelated to death, for example, those associated with microtubule depolymerization, as these still occur under those conditions. ${ }^{48}$ Studies were therefore undertaken to compare the proteome 
and phosphoproteome in cells singly treated with $\mathrm{PCB}$ versus cells doubly treated with PCB and VCR.

First, effects of PCB treatment alone were examined. G1 phase ALL cells were treated with $1 \mu \mathrm{M}$ PCB for $72 \mathrm{~h}$, conditions which induce G1 arrest while maintaining cell viability. ${ }^{48}$ A total of 6,834 proteins were identified from PCBtreated ALL cells compared to vehicle. Unlike VCR treatment, PCB had a profound impact on the proteome, with 1,982 proteins significantly differentiated with an absolute fold change $>2$ and a FDR adjusted $p$-value $<0.05$ (Figure 4A). Because PCB causes a fundamental change in the fate of a G1 phase cell, from one that is cycling and preparing for $S$ phase to a cell that has withdrawn from the cell cycle, a significant redirection of cellular pathways would be anticipated. Indeed, a gene set enrichment analysis of the protein abundance revealed several hallmark pathways, including MTORC1 signaling, Myc targets V1, hypoxia, mitotic spindle, G2M checkpoint, E2F targets, oxidative phosphorylation, and $\mathrm{PI} 3 \mathrm{~K} / \mathrm{AKT} / \mathrm{mTOR}$ signaling pathways as down-regulated, while the p53 pathway and several metabolic pathways were up-regulated (Figure 4B). These findings are in general agreement with other studies which have shown that CDK4/6 inhibitors promote metabolic reprogramming. ${ }^{49}$ The observation of downregulation of E2F targets is fully consistent with the known actions of PCB in inhibiting CDK4/6, a kinase complex which when active phosphorylates retinoblastoma protein, which in turn allows E2F factors to enter the nucleus and transcriptionally upregulate specific target genes. ${ }^{50}$

A total of 1524 phosphosites were identified with 849 phosphopeptides significantly differentiated between PCB and DMSO treatments using the same significance threshold (Figure 4C). Retinoblastoma protein S249 phosphorylation, a known site catalyzed by $\mathrm{CDK} 4 / 6{ }^{50}$ was significantly less abundant after PCB treatment, consistent with expectations (Table S2). Prediction of kinase-substrate relationships was performed (data not shown) which indicated that $\mathrm{PCB}$ caused activation of CSNK2A2 [casein kinase 2 (CK2) catalytic subunit] and inactivation of several kinase pathways including CDK1, CDK2, MAPKAPK2, PRKDC, and DNA-PK. CK2 has a broad specificity and impacts many pathways including those associated with the cell cycle, cell death, and transcriptional regulation, ${ }^{51}$ but its role in the context of G1-arrested ALL cells is presently unclear. Inactivation of CDK pathways was anticipated since arrested cells typically downregulate components of the cell cycle machinery. MAPKAPK2 regulates RNA-binding proteins and transcript stability, ${ }^{52}$ and its inactivation in PCB treated cells likely reflects a lower threshold for transcription since cells have withdrawn from active cycling. Inactivation of PRKDC and DNA-PK, which are involved in DDR (Table 2), likely reflects a reduced requirement for DNA damage surveillance in cells which are withdrawn from the cycle and hence are no longer actively synthesizing or segregating their DNA. Next, ALL cells in G1 phase were pretreated with PCB for $72 \mathrm{~h}$ to induce arrest and then treated with $100 \mathrm{nM}$ VCR or vehicle for $12 \mathrm{~h}$. To confirm that PCB pretreatment blocked the lethal effects of VCR, cell extracts were subjected to immunoblotting for $\gamma \mathrm{H} 2 \mathrm{AX}$ and PARP. As shown in Figure 5A, while VCR treatment alone increased $\gamma \mathrm{H} 2 \mathrm{AX}$ expression and induced PARP cleavage as expected, VCR failed to do so in cells pretreated with PCB.

Corresponding protein samples were subjected to MS analysis. A total of 6833 proteins were identified from the dual treatment study, with 1978 proteins identified as significantly differentiated between dual treatment and vehicle with an absolute fold change $>2$ and FDR-adjusted $p$-value < 0.05 (Figure 5B). A total of 1523 phosphosites were identified with 852 phosphopeptides significantly differentiated (Figure 5C). Similar Hallmark pathways were found to be up and down-regulated in the dual PCB/VCR treatment to those from PCB treatment alone. These include MTORC1 signaling, Myc targets V1, mitotic spindle, G2/M checkpoint, E2F targets, and $\mathrm{PI} 3 \mathrm{~K} / \mathrm{AKT} / \mathrm{MTOR}$ hallmark pathways as down-regulated in the PCB/VCR treatment condition (Figure 5D). These results reinforce results from analysis of single drug treatments, described above, and demonstrate that PCB exerts a much more dominating effect on the proteome and phosphoproteome than VCR, with the majority of those changes occurring independently of VCR.

Comparisons were performed using VENNY, as described in the Experimental Section, to determine signals that were either common or unique to the different treatment conditions. The Venn diagrams in Figure 5D,E show the inter-relationship of the significant proteins and phosphopeptides, respectively, identified from conditions representing VCR versus vehicle, $\mathrm{PCB}$ versus vehicle, and $\mathrm{PCB} / \mathrm{VCR}$ versus vehicle. From the two Venn diagrams, it can be seen that the vast majority of changes in protein and phosphopeptide abundance with drug treatment relative to vehicle was shared between PCB only and the PCB/VCR combination, consistent with data described above. Importantly, this type of analysis allowed us to ask: what protein and phosphopeptide changes are observed in VCR versus vehicle that are not observed in $\mathrm{PCB} / \mathrm{VCR}$ versus vehicle? Since cells die under the first condition but do not under the second condition, such an analysis provided an opportunity to identify signals associated with impending cell death. A single protein, c-Jun, was found significantly and uniquely increased by VCR versus vehicle and was not identified as significant in either of the other treatment conditions, either PCB/VCR dual-treated or treated only with PCB (Figure 5E). Examination of the data in Figure $5 \mathrm{~F}$ indicated there were 62 phosphosites significantly and uniquely increased by VCR treatment alone and not identified as significant in either of the other treatment conditions. One of these was c-Jun S73, confirming the association of JNK/c-Jun in cell death induction. Furthermore, several of the 62 unique signals in Figure $5 \mathrm{~F}$ are represented by proteins with a role in DDR. These included XRCC6 (S560), a helicase required for double-stranded break repair; CDKN2A (S198), a key regulator of the DDR, and Junction-mediating and -regulatory protein (S974), which plays a role in the stress response to DNA damage. ${ }^{44}$ Thus, c-Jun induction, JNK activation, and certain elements of the DDR clearly linked with cell death. Conversely, five phosphoproteins were common to conditions representing VCR versus vehicle and PCB/VCR versus vehicle (Figure $5 \mathrm{~F}$ ) and would therefore not be anticipated to link with cell death. Of these, three play roles in regulation of the cytoskeleton. These included Band 4.1-like protein 1 (S869) and MYO9B (S1342), both of which are involved in actin binding, and CLIP2 (S211) which is involved in microtubule binding, confirming the occurrence of specific signals related to cytoskeletal alterations common to both conditions and not linking to cell death.

\section{CONCLUSIONS}

Quantitative proteomics and phosphoproteomics were used to identify pathways and signaling events associated with cell 
death of G1 phase ALL cells treated with VCR, a MTA that is widely used clinically. ${ }^{4}$ The results revealed distinct alterations associated with JNK signaling, anti-proliferative signaling, the $\mathrm{DDR}$, and cytoskeletal remodeling. While JNK signaling linked to cell death induction, it could be involved in cell survival as a rescue response, since JNK has been implicated in autophagy which is sometimes employed as a salvage pathway. ${ }^{53}$ Further work will be required to determine whether JNK plays a prodeath or anti-death role in the context of VCR-mediated G1 phase death of ALL cells. DDR was also a major response and was shown to be associated with cell death as it was absent in VCR-treated cells that were pretreated with PCB and destined not to die. Our evidence therefore does not support the hypothesis that MTAs may induce death in interphase via DNA damage resulting from defective trafficking of DNA repair proteins, ${ }^{46}$ since this would be expected to occur as a result of microtubule disruption in both cycling and noncycling cells. Rather, DDR in the present context appears to reflect DNA fragmentation associated with the onset of cell death. Finally, our findings revealed actin remodeling as a major response to microtubule depolymerization. Overall, the results suggest potential biomarker candidates to monitor and better understand the mechanisms of MTAs in the clinical setting and indicate that MTAs and actin inhibitors may represent a promising drug combination for ALL that merits further study.

\section{EXPERIMENTAL SECTION}

Reagents. VCR sulfate (SC-201,434) was from Santa Cruz Biotechnology, and palbociclib (PCB) (S1116) was from Selleckchem. Other reagents are as specified.

Cell Culture, Centrifugal Elutriation, and Drug Treatment. Primary ALL-5 cells were maintained in Iscove's Modified Dulbecco's Medium containing $10 \mu \mathrm{g} / \mathrm{mL}$ cholesterol, $6 \mathrm{mg} / \mathrm{mL}$ human serum albumin, $2 \mathrm{mM}$ L-glutamine, $2 \%$ $\mathrm{v} / \mathrm{v}$ amphotericin-B/penicillin/streptomycin, $1 \mu \mathrm{g} / \mathrm{mL}$ insulin, $200 \mu \mathrm{g} / \mathrm{mL} \mathrm{Fe}^{3+}$ saturated human APO-transferrin, and $50 \mu \mathrm{M}$ $\beta$-mercaptoethanol, as described previously, ${ }^{22,33}$ and passaged and utilized up to P20. Cells in G1 phase of the cell cycle were obtained by centrifugal elutriation using a Beckman JE-5.0 elutriation rotor as previously described with slight modifications. $^{22,33}$ Briefly, ALL-5 cells $\left(4-8 \times 10^{8}\right)$ were suspended in $25 \mathrm{~mL}$ of elutriation buffer (Hank's buffered salt solution containing $1.6 \mathrm{~g} / \mathrm{L}$ 2-naphthol-6,8-disulfonic acid dipotassium salt and $2 \%$ fetal bovine serum), passed through a $25 \mathrm{G}$ needle twice and introduced into the elutriation chamber at a flow rate of $25 \mathrm{~mL} / \mathrm{min}$ with a rotor speed of $3000 \mathrm{rpm}$. Rotor speed was reduced to $2920 \mathrm{rpm}$ to collect the F1 wash fraction and then to $2620 \mathrm{rpm}$ to collect the F2 fraction containing cells in G1 phase. The proportion of cells in G1 phase in a typical preparation was $98-99 \%$ as determined by DNA content analysis using propidium iodide staining. ${ }^{33}$ Elutriated G1 phase cells were suspended in growth medium at $1.25 \times 10^{6} / \mathrm{mL}$ and $8 \mathrm{ml}\left(10^{7}\right.$ cells $)$ treated either with vehicle (0.1\% DMSO) or $100 \mathrm{nM}$ VCR for $12 \mathrm{~h}$ in duplicate. The concentration of VCR of $100 \mathrm{nM}$ was previously established as being optimal for inducing death of G1 phase ALL cells. ${ }^{32}$ In some cases, cells were pretreated with the CDK4/6 inhibitor PCB at $1 \mu \mathrm{M}$ for $72 \mathrm{~h}$ prior to treating with vehicle or VCR. After treatment, cells were washed in phosphate-buffered saline, pelleted, flash-frozen in liquid nitrogen, and stored at $-80{ }^{\circ} \mathrm{C}$. Biological triplicate samples from the treatment groups were analyzed by high-resolution mass spectrometry.
Sample Preparation and LC-MS/MS. Cell pellets were lysed in $0.1 \mathrm{~mL}$ of RIPA buffer (Pierce 89900 ) containing a cocktail of both protease inhibitors (Sigma P8340) and phosphatase inhibitors (Pierce A32957). Phosphoproteomics sample preparation followed the same workflow as described in Storey et al. ${ }^{54}$ Briefly, proteins were reduced, alkylated, and digested with sequencing grade trypsin/LysC (Promega VA5071) using Filter-Aided Sample Preparation. The resulting peptides were labeled using a tandem mass tag (TMT) 10-plex isobaric label reagent set (Thermo 90113). The bulk of the peptides $(90 \%)$ were enriched using High-Select $\mathrm{TiO}_{2}$ and $\mathrm{Fe}-$ NTA phosphopeptide enrichment kits (Thermo A32993 and A32992, respectively) following the manufacturer's instructions, while $10 \%$ of the peptides were sequenced for a total proteome analysis. Both enriched and un-enriched labeled peptides were separated into 46 fractions on a $100 \times 1.0 \mathrm{~mm}$ Acquity BEH C18 column (Waters) using an UltiMate 3000 UHPLC system (Thermo) with a 50 min gradient from 99:1 to $60: 40$ buffer $\mathrm{A} / \mathrm{B}$ ratio under basic $\mathrm{pH}$ conditions and then consolidated into 18 super-fractions. Buffer A was $0.1 \%$ formic acid, $0.5 \%$ acetonitrile and buffer B was $0.1 \%$ formic acid, 99.9\% acetonitrile, each adjusted to $\mathrm{pH} 10$ with ammonium hydroxide. Each super-fraction was then further separated by reverse-phase XSelect CSH C18 $2.5 \mu \mathrm{m}$ resin (Waters) on an in-line $150 \times 0.075 \mathrm{~mm}$ column using an UltiMate 3000 RSLCnano system (Thermo). Peptides were eluted using a 60 min gradient from 98:2 to $60: 40$ buffer A/B ratio. Eluted peptides were ionized by electrospray $(2.2 \mathrm{kV})$ followed by mass spectrometric analysis on an Orbitrap Fusion Lumos mass spectrometer (Thermo) using multi-notch MS3 parameters. MS data were acquired using the FTMS analyzer in topspeed profile mode at a resolution of 120,000 over a range of 375 to $1500 \mathrm{~m} / z$. Following CID activation with normalized collision energy of 31.0, MS/MS data were acquired using the ion trap analyzer in centroid mode and normal mass range. Using synchronous precursor selection, up to $10 \mathrm{MS} / \mathrm{MS}$ precursors were selected for HCD activation with normalized collision energy of 55.0, followed by acquisition of MS3 reporter ion data using the FTMS analyzer in the profile mode at a resolution of 50,000 over a range of $100-500 \mathrm{~m} / \mathrm{z}$.

MS Data Analysis. Proteins were identified, and TMT MS3 reporter ions were quantified by searching the UniprotKB Homo sapiens database (June 2018) using MaxQuant (version 1.6.0.16, Max Planck Institute) with a parent ion tolerance of 3 $\mathrm{ppm}$, a fragment ion tolerance of $0.5 \mathrm{Da}$, a reporter ion tolerance of $0.001 \mathrm{Da}$, trypsin/P enzyme with two missed cleavages, variable modifications including oxidation on $\mathrm{M}$, acetyl on protein $\mathrm{N}$-terminus, phosphorylation on STY, and fixed modification of carbamidomethyl on C. Protein and peptide identifications were accepted if they could be established with less than $1.0 \%$ false discovery. TMT MS3corrected reporter ion intensity values were analyzed for changes in total protein using the unenriched lysate samples. Phospho(STY) modifications were analyzed using the samples enriched for phosphorylated peptides. The enriched and unenriched samples were multiplexed using two separate TMT10-plex batches, one for the enriched and one for the un-enriched samples.

Following data acquisition and database search, the search results were normalized and sample quality was verified using ProteiNorm, a user-friendly tool for a systematic evaluation of normalization methods, imputation of missing values, and comparisons of different differential abundance methods. ${ }^{55}$ 
The protein and phosphopeptide data were normalized using cyclic loss. ${ }^{56}$ Linear models were then fitted to the expression data using Linear Models for Microarray Data (limma 3.46.0) ImFit function, and differential abundance was evaluated using robust empirical Bayes (eBayes) to protect against hypervariable and hypo-variable proteins/phosphopeptides. ${ }^{54,57}$ Proteins and phosphopeptides with an absolute fold-change $>2$ and a FDR adjusted $p$-value $<0.05$ were considered significant. ${ }^{58}$ The phosphosites were filtered to retain only peptides with a localization probability $>75 \%$, filter peptides with zero values, and $\log 2$ transformed prior to differential abundance analysis with limma. Gene set enrichment analysis for the protein data set was performed using Ensemble of Gene Set Enrichment Analyses to identify key pathways. ${ }^{59,60}$ Modified phosphosite-flanking peptides were evaluated using PHOXTRACK to identify kinases and their substrates. ${ }^{61}$ The 13-mer phosphosite flanking peptides ( \pm 6 amino acids) and corresponding $\log _{2}$ fold-changes obtained for each comparison were used as input. Kinase activity/enrichment of known kinase substrates was assessed using 50,000 permutations, requiring a minimum number of three substrates, unweighted statistics.

Immunoblot Analysis. Whole cell extracts were prepared in the presence of protease and phosphatase inhibitors as previously described, ${ }^{62}$ and samples $(25 \mu \mathrm{g}$ protein per lane) were analyzed by sodium dodecyl sulfate-polyacrylamide gel electrophoresis using $12 \%$ acrylamide gels followed by transfer to a PVDF membrane. Membranes were probed with the following antibodies: poly(ADP-ribose) PARP (Cell Signaling \#9532, 1:2500 dilution); c-Jun (BD Biosciences \#610326, 1:1000 dilution); phospho(S73)-c-Jun (Santa Cruz \#SC-822, 1:1000 dilution); $\gamma \mathrm{H} 2 \mathrm{AX}$ (Cell Signaling 9718, 1:1,000 dilution); and GAPDH (Cell Signaling \#2118, 1:10,000 dilution). Molecular mass determinations were estimated based on the use of pre-stained standard proteins from GE Healthcare, Product \# RPN800E.

\section{ASSOCIATED CONTENT}

\section{(s) Supporting Information}

The Supporting Information is available free of charge at https://pubs.acs.org/doi/10.1021/acsomega.1c03936.

Ten of the significantly differentiating phosphopeptides, PHOXTRACK analysis of significant phosphopeptides, and mass spectrometry proteomics data deposited to the ProteomeXchange Consortium via the PRIDE ${ }^{63}$ partner repository with the data set identifier PXD027190 and 10.6019/PXD027190 (XLSX)

\section{AUTHOR INFORMATION}

\section{Corresponding Authors}

Stephanie D. Byrum - Department of Biochemistry and Molecular Biology, University of Arkansas for Medical Sciences, Little Rock, Arkansas 72205, United States; Arkansas Children's Research Institute, Little Rock, Arkansas 72202, United States; Winthrop P. Rockefeller Cancer Institute, Little Rock, Arkansas 72205, United States; (1) orcid.org/0000-0002-1783-3610; Phone: (501) 603-1224; Email: sbyrum@uams.edu

Timothy C. Chambers - Department of Biochemistry and Molecular Biology, University of Arkansas for Medical Sciences, Little Rock, Arkansas 72205, United States; Winthrop P. Rockefeller Cancer Institute, Little Rock,
Arkansas 72205, United States; 이잉.org/0000-00023122-9794; Phone: (501) 686-5755;

Email: chamberstimothyc@uams.edu

\section{Authors}

Magdalena Delgado - Department of Biochemistry and Molecular Biology, University of Arkansas for Medical Sciences, Little Rock, Arkansas 72205, United States; Present Address: Department of Pharmacology, UTSW Medical Center, 5323 Harry Hines Boulevard, Dallas, TX 75390

Charity L. Washam - Department of Biochemistry and Molecular Biology, University of Arkansas for Medical Sciences, Little Rock, Arkansas 72205, United States; Arkansas Children's Research Institute, Little Rock, Arkansas 72202, United States

Alicja Urbaniak - Department of Biochemistry and Molecular Biology, University of Arkansas for Medical Sciences, Little Rock, Arkansas 72205, United States

Billie Heflin - Department of Biochemistry and Molecular Biology, University of Arkansas for Medical Sciences, Little Rock, Arkansas 72205, United States

Aaron J. Storey - Department of Biochemistry and Molecular Biology, University of Arkansas for Medical Sciences, Little Rock, Arkansas 72205, United States

Renny S. Lan - Department of Biochemistry and Molecular Biology, University of Arkansas for Medical Sciences, Little Rock, Arkansas 72205, United States

Samuel G. Mackintosh - Department of Biochemistry and Molecular Biology, University of Arkansas for Medical Sciences, Little Rock, Arkansas 72205, United States

Alan J. Tackett - Department of Biochemistry and Molecular Biology, University of Arkansas for Medical Sciences, Little Rock, Arkansas 72205, United States; Arkansas Children's Research Institute, Little Rock, Arkansas 72202, United States; Winthrop P. Rockefeller Cancer Institute, Little Rock, Arkansas 72205, United States; (1) orcid.org/0000-0002$3672-4460$

Complete contact information is available at: https://pubs.acs.org/10.1021/acsomega.1c03936

\section{Notes}

The authors declare no competing financial interest.

\section{ACKNOWLEDGMENTS}

This work was supported by National Institutes of Health grants P20GM121293 (AJT) and R24GM137786 (AJT) and by funds from the Winthrop P. Rockefeller Cancer Institute, UAMS (TCC). We thank Dr. Duah Alkam for assistance with the Table of Contents Graphic.

\section{REFERENCES}

(1) Steinmetz, M. O.; Prota, A. E. Microtubule-Targeting Agents: Strategies To Hijack the Cytoskeleton. Trends Cell Biol. 2018, 28, $776-792$.

(2) Dumontet, C.; Jordan, M. A. Microtubule-binding agents: a dynamic field of cancer therapeutics. Nat. Rev. Drug Discov. 2010, 9, 790-803.

(3) Rowinsky, E. K.; Donehower, R. C. The Chemotherapy Source Book; Williams and Wilkins: Baltimore, MD, 1998; pp 387-423.

(4) Bates, D.; Eastman, A. Microtubule destabilising agents: far more than just antimitotic anticancer drugs. Br. J. Clin. Pharmacol. 2017, 83, $255-268$.

(5) Martino, E.; Casamassima, G.; Castiglione, S.; Cellupica, E.; Pantalone, S.; Papagni, F.; Rui, M.; Siciliano, A. M.; Collina, S. Vinca 
alkaloids and analogues as anti-cancer agents: Looking back, peering ahead. Bioorg. Med. Chem. Lett. 2018, 28, 2816-2826.

(6) Yang, C.-P. H.; Horwitz, S. B. Taxol((R)): The First Microtubule Stabilizing Agent. Int. J. Mol. Sci. 2017, 18, 1733.

(7) Prota, A. E.; Bargsten, K.; Zurwerra, D.; Field, J. J.; Díaz, J. F.; Altmann, K.-H.; Steinmetz, M. O. Molecular mechanism of action of microtubule-stabilizing anticancer agents. Science 2013, 339, 587590.

(8) Jordan, M. A.; Wilson, L. Microtubules as a target for anticancer drugs. Nat. Rev. Canc. 2004, 4, 253-265.

(9) Rieder, C. L.; Maiato, H. Stuck in division or passing through: what happens when cells cannot satisfy the spindle assembly checkpoint. Dev. Cell 2004, 7, 637-651.

(10) Weaver, B. A. A.; Cleveland, D. W. Decoding the links between mitosis, cancer, and chemotherapy: The mitotic checkpoint, adaptation, and cell death. Canc. Cell 2005, 8, 7-12.

(11) Yamada, H. Y.; Gorbsky, G. J. Spindle checkpoint function and cellular sensitivity to antimitotic drugs. Mol. Canc. Therapeut. 2006, 5, 2963-2969.

(12) Manchado, E.; Guillamot, M.; Malumbres, M. Killing cells by targeting mitosis. Cell Death Differ. 2012, 19, 369-377.

(13) Topham, C. H.; Taylor, S. S. Mitosis and apoptosis: how is the balance set? Curr. Opin. Cell Biol. 2013, 25, 780-785.

(14) Miltenburg, N. C.; Boogerd, W. Chemotherapy-induced neuropathy: A comprehensive survey. Cancer Treat. Rev. 2014, 40, $872-882$.

(15) Rowinsky, E. K.; Eisenhauer, E. A.; Chaudhry, V.; Arbuck, S. G.; Donehower, R. C. Clinical toxicities encountered with paclitaxel (Taxol). Semin. Oncol. 1993, 20, 1-15.

(16) Carlson, K.; Ocean, A. J. Peripheral neuropathy with microtubule-targeting agents: occurrence and management approach. Clin. Breast Cancer 2011, 11, 73-81.

(17) Jackson, J. R.; Patrick, D. R.; Dar, M. M.; Huang, P. S. Targeted anti-mitotic therapies: can we improve on tubulin agents? Nat. Rev. Canc. 2007, 7, 107-117.

(18) Komlodi-Pasztor, E.; Sackett, D. L.; Fojo, T. Tales of how great drugs were brought down by a flawed rationale-response. Clin. Cancer Res. 2013, 19, 1304.

(19) Yan, V. C.; Butterfield, H. E.; Poral, A. H.; Yan, M. J.; Yang, K. L.; Pham, C.-D.; Muller, F. L. Why Great Mitotic Inhibitors Make Poor Cancer Drugs. Trends Cancer 2020, 6, 924-941.

(20) Fürst, R.; Vollmar, A. M. A new perspective on old drugs: nonmitotic actions of tubulin-binding drugs play a major role in cancer treatment. Pharmazie 2013, 68, 478-483.

(21) Mitchison, T. J. The proliferation rate paradox in antimitotic chemotherapy. Mol. Biol. Cell 2012, 23, 1-6.

(22) Kothari, A.; Hittelman, W. N.; Chambers, T. C. Cell CycleDependent Mechanisms Underlie Vincristine-Induced Death of Primary Acute Lymphoblastic Leukemia Cells. Cancer Res. 2016, 76, $3553-3561$.

(23) Gascoigne, K. E.; Taylor, S. S. How do anti-mitotic drugs kill cancer cells? J. Cell Sci. 2009, 122, 2579-2585.

(24) Huang, H.-C.; Shi, J.; Orth, J. D.; Mitchison, T. J. Evidence that mitotic exit is a better cancer therapeutic target than spindle assembly. Canc. Cell 2009, 16, 347-358.

(25) Sinha, D.; Duiff, P. H. G.; Khanna, K. K. Mitotic slippage: an old tale with a new twist. Cell Cycle 2019, 18, 7-15.

(26) Terrano, D. T.; Upreti, M.; Chambers, T. C. Cyclin-dependent kinase 1-mediated $\mathrm{Bcl}-\mathrm{xL} / \mathrm{Bcl}-2$ phosphorylation acts as a functional link coupling mitotic arrest and apoptosis. Mol. Cell Biol. 2010, 30, 640-656.

(27) Eichhorn, J. M.; Sakurikar, N.; Alford, S. E.; Chu, R.; Chambers, T. C. Critical role of anti-apoptotic Bcl-2 protein phosphorylation in mitotic death. Cell Death Dis. 2013, 4, No. e834. (28) Sakurikar, N.; Eichhorn, J. M.; Alford, S. E.; Chambers, T. C. Identification of a mitotic death signature in cancer cell lines. Canc. Lett. 2014, 343, 232-238.

(29) Manning, B. D.; Toker, A. AKT/PKB Signaling: Navigating the Network. Cell 2017, 169, 381-405.
(30) Clarke, P. R.; Allan, L. A.; Skowyra, A. Timed degradation of Mcl-1 controls mitotic cell death. Mol. Cell. Oncol. 2018, 5, No. e1516450.

(31) Gong, Y.; Fan, Z.; Luo, G.; Yang, C.; Huang, Q.; Fan, K.; Cheng, H.; Jin, K.; Ni, Q.; Yu, X.; Liu, C. The role of necroptosis in cancer biology and therapy. Mol. Cancer 2019, 18, 100.

(32) Delgado, M.; Urbaniak, A.; Chambers, T. C. Contrasting effects of microtubule destabilizers versus stabilizers on induction of death in G1 phase of the cell cycle. Biochem. Pharmacol. 2019, 162, 213-223.

(33) Delgado, M.; Kothari, A.; Hittelman, W. N.; Chambers, T. C. Preparation of Primary Acute Lymphoblastic Leukemia Cells in Different Cell Cycle Phases by Centrifugal Elutriation. J. Visualized Exp. 2017, 129, 56418.

(34) Mah, L.-J.; El-Osta, A.; Karagiannis, T. C. $\gamma \mathrm{H} 2 \mathrm{AX}$ : a sensitive molecular marker of DNA damage and repair. Leukemia 2010, 24, 679-686.

(35) Karin, M.; Gallagher, E. From JNK to pay dirt: jun kinases, their biochemistry, physiology and clinical importance. IUBMB Life 2005, 57, 283-295.

(36) Angel, P.; Hattori, K.; Smeal, T.; Karin, M. The jun protooncogene is positively autoregulated by its product, Jun/AP-1. Cell $1988,55,875-885$.

(37) Berry, A.; Goodwin, M.; Moran, C. L.; Chambers, T. C. AP-1 activation and altered AP-1 composition in association with increased phosphorylation and expression of specific Jun and Fos family proteins induced by vinblastine in KB-3 cells. Biochem. Pharmacol. 2001, 62, 581-591.

(38) Pearson, G.; Robinson, F.; Beers Gibson, T.; Xu, B.-e.; Karandikar, M.; Berman, K.; Cobb, M. H. Mitogen-activated protein (MAP) kinase pathways: regulation and physiological functions. Endocr. Rev. 2001, 22, 153-183.

(39) Alarcon-Vargas, D.; Ronai, Z. e. c-Jun-NH2 kinase (JNK) contributes to the regulation of c-Myc protein stability. J. Biol. Chem. 2004, 279, 5008-5016.

(40) Bodakuntla, S.; Jijumon, A. S.; Villablanca, C.; GonzalezBillault, C.; Janke, C. Microtubule-Associated Proteins: Structuring the Cytoskeleton. Trends Cell Biol. 2019, 29, 804-819.

(41) Zhu, Y.; Kakinuma, N.; Wang, Y.; Kiyama, R. Kank proteins: a new family of ankyrin-repeat domain-containing proteins. Biochim. Biophys. Acta 2008, 1780, 128-133.

(42) Grintsevich, E. E. Remodeling of Actin Filaments by Drebrin A and Its Implications. Adv. Exp. Med. Biol. 2017, 1006, 61-82.

(43) Stehn, J. R.; Haass, N. K.; Bonello, T.; Desouza, M.; Kottyan, G.; Treutlein, H.; Zeng, J.; Nascimento, P. R. B. B.; Sequeira, V. B.; Butler, T. L.; Allanson, M.; Fath, T.; Hill, T. A.; McCluskey, A.; Schevzov, G.; Palmer, S. J.; Hardeman, E. C.; Winlaw, D.; Reeve, V. E.; Dixon, I.; Weninger, W.; Cripe, T. P.; Gunning, P. W. A novel class of anticancer compounds targets the actin cytoskeleton in tumor cells. Cancer Res. 2013, 73, 5169-5182.

(44) Andres-Leon, E.; Cases, I.; Arcas, A.; Rojas, A. M. DDRprot: a database of DNA damage response-related proteins. Database 2016, 2016, baw123.

(45) Salerni, B. L.; Bates, D. J.; Albershardt, T. C.; Lowrey, C. H.; Eastman, A. Vinblastine induces acute, cell cycle phase-independent apoptosis in some leukemias and lymphomas and can induce acute apoptosis in others when Mcl-1 is suppressed. Mol. Canc. Therapeut. 2010, 9, 791-802.

(46) Poruchynsky, M. S.; Komlodi-Pasztor, E.; Trostel, S.; Wilkerson, J.; Regairaz, M.; Pommier, Y.; Zhang, X.; Kumar Maity, T.; Robey, R.; Burotto, M.; Sackett, D.; Guha, U.; Fojo, A. T. Microtubule-targeting agents augment the toxicity of DNA-damaging agents by disrupting intracellular trafficking of DNA repair proteins. Proc. Natl. Acad. Sci. U.S.A. 2015, 112, 1571-1576.

(47) Toogood, P. L.; Harvey, P. J.; Repine, J. T.; Sheehan, D. J.; VanderWel, S. N.; Zhou, H.; Keller, P. R.; McNamara, D. J.; Sherry, D.; Zhu, T.; Brodfuehrer, J.; Choi, C.; Barvian, M. R.; Fry, D. W. Discovery of a potent and selective inhibitor of cyclin-dependent kinase 4/6. J. Med. Chem. 2005, 48, 2388-2406. 
(48) Delgado, M.; Chambers, T. C. Microtubules play an essential role in the survival of primary acute lymphoblastic leukemia cells advancing through G1 phase. Cell Cycle 2018, 17, 1784-1796.

(49) Franco, J.; Balaji, U.; Freinkman, E.; Witkiewicz, A. K.; Knudsen, E. S. Metabolic Reprogramming of Pancreatic Cancer Mediated by CDK4/6 Inhibition Elicits Unique Vulnerabilities. Cell Rep. 2016, 14, 979-990.

(50) Weinberg, R. A. The retinoblastoma protein and cell cycle control. Cell 1995, 81, 323-330.

(51) Borgo, C.; D’Amore, C.; Sarno, S.; Salvi, M.; Ruzzene, M. Protein kinase CK2: a potential therapeutic target for diverse human diseases. Signal Transduction Targeted Ther. 2021, 6, 183.

(52) Soni, S.; Anand, P.; Padwad, Y. S. MAPKAPK2: the master regulator of RNA-binding proteins modulates transcript stability and tumor progression. J. Exp. Clin. Cancer Res. 2019, 38, 121.

(53) Wu, Q.; Wu, W.; Fu, B.; Shi, L.; Wang, X.; Kuca, K. JNK signaling in cancer cell survival. Med. Res. Rev. 2019, 39, 2082-2104.

(54) Storey, A. J.; Naceanceno, K. S.; Lan, R. S.; Washam, C. L.; Orr, L. M.; Mackintosh, S. G.; Tackett, A. J.; Edmondson, R. D.; Wang, Z.; Li, H.-y.; Frett, B.; Kendrick, S.; Byrum, S. D. ProteoViz: a tool for the analysis and interactive visualization of phosphoproteomics data. Mol. Omics 2020, 16, 316-326.

(55) Graw, S.; Tang, J.; Zafar, M. K.; Byrd, A. K.; Bolden, C.; Peterson, E. C.; Byrum, S. D. proteiNorm - A User-Friendly Tool for Normalization and Analysis of TMT and Label-Free Protein Quantification. ACS Omega 2020, 5, 25625-25633.

(56) Ritchie, M. E.; Phipson, B.; Wu, D.; Hu, Y.; Law, C. W.; Shi, W.; Smyth, G. K. limma powers differential expression analyses for RNA-sequencing and microarray studies. Nucleic Acids Res. 2015, 43, No. e47.

(57) Phipson, B.; Lee, S.; Majewski, I. J.; Alexander, W. S.; Smyth, G. K. Robust Hyperparameter Estimation Protects against Hypervariable Genes and Improves Power to Detect Differential Expression. Ann. Appl. Stat. 2016, 10, 946-963.

(58) Benjamini, Y.; Hochberg, Y. Controlling the false discovery rate; A practical and powerful approach to multiple testing. J. $R$. Stat. Soc., B 1995, 57, 289-300.

(59) Alhamdoosh, M.; Ng, M.; Wilson, N. J.; Sheridan, J. M.; Huynh, H.; Wilson, M. J.; Ritchie, M. E. Combining multiple tools outperforms individual methods in gene set enrichment analyses. Bioinformatics 2017, 33, 414-424.

(60) Alhamdoosh, M.; Law, C. W.; Tian, L.; Sheridan, J. M.; Ng, M.; Ritchie, M. E. Easy and efficient ensemble gene set testing with EGSEA. F1000Research 2017, 6, 2010.

(61) Weidner, C.; Fischer, C.; Sauer, S. PHOXTRACK-a tool for interpreting comprehensive datasets of post-translational modifications of proteins. Bioinformatics 2014, 30, 3410-3411.

(62) Fan, M.; Du, L.; Stone, A. A.; Gilbert, K. M.; Chambers, T. C. Modulation of mitogen-activated protein kinases and phosphorylation of Bcl-2 by vinblastine represent persistent forms of normal fluctuations at G2-M1. Cancer Res. 2000, 60, 6403-6407.

(63) Perez-Riverol, Y.; Csordas, A.; Bai, J.; Bernal-Llinares, M.; Hewapathirana, S.; Kundu, D. J.; Inuganti, A.; Griss, J.; Mayer, G.; Eisenacher, M.; Pérez, E.; Uszkoreit, J.; Pfeuffer, J.; Sachsenberg, T.; Yılmaz, Ş.; Tiwary, S.; Cox, J.; Audain, E.; Walzer, M.; Jarnuczak, A. F.; Ternent, T.; Brazma, A.; Vizcaíno, J. A. The PRIDE database and related tools and resources in 2019: improving support for quantification data. Nucleic Acids Res. 2019, 47, D442-D450. 